Results: Among the 541 patients screened, hypertension was present in $28.1 \%$ patients, dyslipidemia in $19.2 \%$, chronic respiratory tract diseases in $12.8 \%$ and diabetes in $9.6 \%$. Screening led to the following recommendations: blood pressure monitoring (22.6\% patients), dietary advice $(56.8 \%)$, cardiologist referral $(35.5 \%)$, intensification of physical activity $(27.0 \%)$, cancer screening $(50.5 \%)$, vaccinations $(60.6 \%)$ and vitamino-calcium supplementation $(30.3 \%)$. On the 237 patients called back, $72.3 \%$ underwent blood pressure monitoring, $58.6 \%$ followed dietary advice, $64.4 \%$ took vitamino-calcium supplementation, $55.2 \%$ had vaccinations done, $52.1 \%$ saw a cardiologist, $42.7 \%$ increased physical activity and $31.4 \%$ performed cancer screening. No specific gender, age, pathology, or psychological factors were associated with adherence to recommendations.

Conclusions: This study underlines the relevance of a systematic screening of multimorbidities in chronic inflammatory rheumatic diseases, and the good patient's adherence rate to the recommendations.

Disclosure of Interest: None declared

DOI: 10.1136/annrheumdis-2018-eular.3756

\section{AB1258 EARLY ARTHRITIS SERVICE IS COST EFFECTIVE, IMPROVES OUTCOMES AND REDUCES BIOLOGIC USE}

G.A. Niazi, M.K. Nisar. Rheumatology, Luton and Dunstable University Hospital, Luton, UK

Background: There is good evidence that dedicated early arthritis clinics (EACs) improve referral lag time and reduce delay in establishing disease-modifying therapy. However it remains arguable whether such clinics improve relevant disease outcomes. Nationally, only $57 \%$ of units have dedicated EACs.

Objectives: We established Early Arthritis Service (EAS), centred on NICE RA quality standards, to reduce the time to diagnosis and the start of definitive therapy with an aim to accomplish good outcomes by the introduction of dedicated Early Arthritis Clinics (EACs).

Methods: The department set up an early arthritis service with introduction of six clinics (EACs) every week. An agreed treatment protocol incorporating ultrasound was developed to ensure standardised approach to early initiation of treatment, drug education and timely review. This is a retrospective study of all patients presenting to the service in the first year.

Results: Our catchment area covers a population of 350000 with $40 \%$ ethnic minorities. Of 1884 patients referred, 482 (25.5\%) were triaged into EACs based on set criteria. All were reviewed within 3 weeks. 247 (51\%) were confirmed to have early arthritis. Mean age was 52.4 years (17-86y). 157 (63.5\%) were women. 177 $(71.6 \%)$ were White, $58(23.5 \%)$ of Asian and twelve of other background. 159 $(64.3 \%)$ had RA, 57 (23\%) with PsA and 31 had other inflammatory arthritides. 25 $(10 \%)$ had erosions at presentation. There was median 26 weeks delay (0.41043 weeks) from symptom onset to GP presentation. Median time for GP referral to the department was 4.0 days (0-84 days). Mean DAS28 at first visit was 4.65 $(0.6-8.0, \mathrm{n}=166)$

$95 \%$ commenced their DMARDs within 3 week of initial review. Other $5 \%$ who missed the target was owing to patient factors. Treating to target achieved DAS28 remission for $84(53.5 \%)$ and low disease activity for a further $44(34 \%)$. Median time to achieve remission or LDA was 20 weeks ( $0-52$ weeks, $n=128)$. Similarly, $40 / 57(70 \%)$ of PsA patients achieved good PsARC response in median 24 weeks. Of 247 , only 21 ( $8.5 \%$ ) patients required escalation to biologic therapy. Conclusions: Dedicated EACs help achieve good clinical outcomes in majority of patients. Nearly $87 \%$ of our cohort attained remission or low disease activity in less than six months. This was despite a significant delay in patients presenting to their GPs and moderately-high disease activity. $100 \%$ of our patients were treated to target facilitated by protocol driven escalation of therapy in these clinics. This is in contrast to the national audit findings whereby only $68 \%$ of patients were treated with disease modifying drugs within 6 weeks of referral and $89 \%$ had treatment to target. Patient experience also improved ( $94 \%$ would now recommend the service compared to $76 \%$ prior to the initiative).

The project was a financial success with total savings for the year, accounting for most generous cost estimates, were $£ 136$ 973. In addition, there was a $42 \%$ reduction in biologic use in this group compared to 2015 . These savings are on top of wider economic and societal benefits achieved by inducing low disease activity or remission.

Disclosure of Interest: None declared

DOI: 10.1136/annrheumdis-2018-eular.1329

\section{AB1259 IS DOSE BANDING OF INFLIXIMAB COST EFFECTIVE - RESULTS FROM REAL WORLD IMPLEMENTATION}

\section{M.K. Nisar. Rheumatology, Luton and Dunstable University Hospital, Luton, UK}

Background: Infliximab dose is based on the patient's body weight (3-5 mg/kg) and requires the use of multiple $100 \mathrm{mg}$ vials to reconstitute the individualised dose. As the product is expensive, there is a significant cost associated with unused and wasted infliximab in any part-used vials. "Dose banding" is one solu tion so that patients receive a pre-prepared dose produced in advance in batches where wastage from part vials is minimised. There is some encouraging data that reconstituted infliximab is stable for long enough to allow batch production of preprepared doses. The theoretical risk of course is over or under treatment while respectively rounding up or down the administered dose.

Objectives: We undertook a retrospective analysis of our experience of dose banding in rheumatology unit.

Methods: All patients prescribed infliximab for rheumatic indication at our centre were included in the analysis. Case notes were retrospectively reviewed to look a dose variance following implementation of this initiative, resultant disease contro and the consequent cost savings.

Results: 10 patients prescribed infliximab biosimilar and two bio-originator were identified for the analysis. Median age was 55.5 years (range $25-80$ years). Nine had the drug for RA, two for AS and one for myositis.

Three had no change in dose as a result of implementing dose banding program. Three had dose increased by $3 \%-5 \%$. Remaining six had dose reduced by range of $4 \%-6 \%$. Their disease scores before and after dose banding remained largely unchanged (table 1). This equated to $£ 1,184.58$ annual savings in addition to the economic benefit of successfully switching the ten patients to biosimilar. No patient suffered loss of efficacy or adverse events in any of the three groups.

Conclusions: Our data confirms the utility of dose banding program for infliximab in rheumatology practice with no concerns about efficacy or safety of the initiative. There are potential opportunities to achieve considerable reductions in medicines discarded and improving finances by adopting dose banding for infliximab. It also helped curb over $£ 10000$ expense to our Trust in infliximab waste cost alone. There are some important caveats to consider as well. True savings will depend on list price of each product and varies with originator versus biosimilar. The variance increases as the width of the dose bands increases i.e. the variance is greater at lower body weights. Consequently, depending on patient population both clinical and cost considerations could alter. Finally, assurance of the product

Abstract AB1259 - Table 1

\begin{tabular}{|c|c|c|c|c|c|c|c|c|c|c|}
\hline Age & Sex & Ethnicity & Indication & $\begin{array}{l}\text { Prior dose } \\
\quad(\mathrm{mg})\end{array}$ & $\begin{array}{l}\text { Pre banding } \\
\text { Disease } \\
\text { score }\end{array}$ & $\begin{array}{c}\text { New dose } \\
(\mathrm{mg})\end{array}$ & $\begin{array}{l}\text { Post banding } \\
\text { Disease } \\
\text { score }\end{array}$ & Variance & $\begin{array}{l}\text { Dose } \\
\text { vials }\end{array}$ & $\begin{array}{c}\text { Saving } \\
\text { (12 month) }\end{array}$ \\
\hline 48 & $M$ & Mixed & AS & 400 & 3.2 & 400 & 3.9 & $0 \%$ & 4 & $£ 0.00$ \\
\hline 58 & $\mathrm{~F}$ & White & RA & 210 & 2.3 & 200 & 2.8 & $-5 \%$ & 2 & $£ 87.00$ \\
\hline 54 & $\mathrm{~F}$ & White & RA & 300 & 3.6 & 300 & 1.7 & $0 \%$ & 3 & $£ 0.00$ \\
\hline 44 & $\mathrm{~F}$ & White & RA & 210 & 2.2 & 200 & 1.8 & $-5 \%$ & 3 & $£ 87.00$ \\
\hline 78 & $\mathrm{~F}$ & Asian & RA & 170 & 4.1 & 175 & 3.3 & $3 \%$ & 2 & $-£ 43.50$ \\
\hline 56 & $\mathrm{~F}$ & White & RA & 300 & 2.5 & 300 & 2.1 & $0 \%$ & 2 & $£ 0.00$ \\
\hline 73 & $\mathrm{~F}$ & White & RA & 210 & 3.9 & 200 & 3.9 & $-5 \%$ & 2 & $£ 87.00$ \\
\hline 80 & $\mathrm{~F}$ & White & RA & 190 & 3.8 & 200 & 3.8 & $5 \%$ & 3 & $-£ 87.00$ \\
\hline 49 & $\mathrm{~F}$ & White & RA & 210 & 2.3 & 200 & 3.4 & $-5 \%$ & 3 & $£ 87.00$ \\
\hline 55 & $M$ & White & AS & 320 & 0.2 & 300 & 0.4 & $-6 \%$ & 2 & $£ 174.00$ \\
\hline \multicolumn{11}{|c|}{ Bio-originator } \\
\hline \multicolumn{11}{|c|}{ Patients } \\
\hline 25 & $\mathrm{~F}$ & White & RA & 120 & 4.1 & 125 & 4.1 & $4 \%$ & 0 & $£ 0.00$ \\
\hline 49 & $\mathrm{~F}$ & White & Myositis & 470 & $\begin{array}{c}\text { Well } \\
\text { controlled }\end{array}$ & 450 & $\begin{array}{c}\text { Well } \\
\text { controlled }\end{array}$ & $-4 \%$ & 0 & $£ 793.08$ \\
\hline
\end{tabular}


integrity and stability is reliant on efficient pharmacy processes necessary to accommodate the use of new formulations for dose banding.

Disclosure of Interest: None declared

DOI: 10.1136/annrheumdis-2018-eular.1300

\section{AB1260 BASELINE CHARACTERISTICS AND PATIENT SATISFACTION DATA FROM COACH@HOME: THE GERMAN SUPPORT PROGRAM FOR PATIENTS WITH RHEUMATIC DISEASES TREATED WITH CERTOLIZUMAB PEGOL}

N. Böhme ${ }^{1}$, A.-D. Holst ${ }^{2}$, F. Dybowski ${ }^{3}$, C. Volberg ${ }^{4}$, H.-G. Pott ${ }^{5}$, U. Lendl ${ }^{6}$. ${ }^{1}$ Healthcare at Home Deutschland $\mathrm{GmbH}$, Weinheim; ${ }^{2}$ Rheumapraxis, Ludwigslust, ${ }^{3}$ Rheumapraxis Ruhr, Herne; ${ }^{4}$ Rheumatologie in Neuss, Neuss; ${ }^{5}$ Rheumatologikum Hannover, Hannover, ${ }^{6}$ UCB Pharma, Monheim, Germany

Background: Certolizumab pegol (CZP)-treated patients (pts) with rheumatoid arthritis (RA), axial spondyloarthritis (axSpA, including ankylosing spondylitis [AS] and non-radiographic [nr-] $\mathrm{axSpA}$ ), and psoriatic arthritis (PsA) may be recommended the patient-coaching program, coach@home (c@h) in Germany to manage their disease. Eight coaching calls are offered over one year, scheduled around 'critical' milestones along the therapeutic journey. These calls are made by professionally-trained nurses, who offer support and information about both the disease and its treatment with CZP

Objectives: The purpose of this observational analysis was to assess the baseline characteristics of pts subscribed to c@h, and the level of pt satisfaction with the program.

Methods: c@h was launched in ,June 2014 and is available to pts who are prescribed CZP according to the local product label. Pts must be CZP-naïve when subscribed to the program, and pt consent is required prior to subscription by the treating physician. There are no additional criteria for enrolment, although the program must be recommended to the pt by the treating physician.

Pt satisfaction was measured either at the end of the program or at discontinuation using the net promoter score (NPS), which has previously been used for this purpose. ${ }^{1}$ The NPS is derived by asking pts to state how likely it is that they would recommend c@h to others (on a 0-10 scale). Rankings of 9-10 are considered 'promoters', 7-8 'passives', and 0-6 'detractors'. Subtracting the percentage of detractors from that of promoters yields the NPS (figure 1).

Results: A cumulative total of 655 pts had been registered to the program as of 5 .ct 2017 Of the total number of reported indications $(n=683$; multiple indications per pt possible), 55.9\% were RA, $16.5 \%$ AS, $7.6 \% \mathrm{nr}$-axSpA, and $19.9 \%$ PsA. The mean age at baseline was 55 (RA), 54 (AS), 53 (nr-axSpA), and 52 (PsA), and the proportion of female pts was $80 \%, 58 \%, 65 \%$, and $69 \%$, respectively. Prior biologic DMARD exposure was $32.8 \%$.

The most common topics discussed on phone calls were therapy compliance (in 1339 calls), CZP maintenance dose (in 1333 calls) and syringe disposal (in 870 calls). The average length per call was $15.9 \mathrm{~min}$ at the end of Week 0 (total: 28 calls), and $14.0 \mathrm{~min}$ at the end of Week 52 (total: 1796 calls). A cumulative total of 272 pts had either completed the 1 year coaching period $(n=70)$ or discontinued $(n=202)$ by 28 , Sep 2017 of whom 106 rated the program. Of these, $87.5 \%$ gave promoter scores, $10.4 \%$ passive, and $3.8 \%$ detractor, yielding an NPS of 83.7 (figure 1). The program was still ongoing at the time of this data cut.
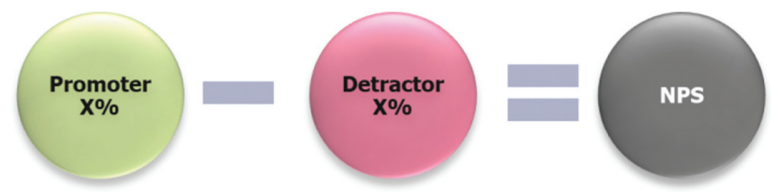

口9-10: Promoter

口7-8: Passive

口0-6: Detractor

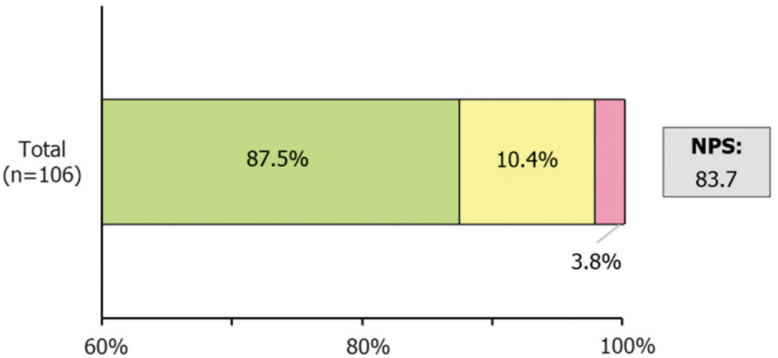

Abstract AB1260 - Figure 1. Patient ratings and Net Promoter Score for the coach@home program (as of September 2017)
Patients were asked to rate the coach@home program on a scale of 0-10, where $0=$ "I would certainly not recommend this program" and 10="I would most probably recommend this program". Scores of 9-10 were considered 'promoters', 7-8 'pas sives' and 0-6 'detractors'. Percentages shown are out of the total number of responders by the end of September 2017 ( $n=106)$. NPS: Net Promoter Score.

Conclusions: The c@h program offers guidance and support to pts treated with CZP in Germany. Feedback from pts who were willing to provide a rating indicates a high level of satisfaction with the program.

\section{REFERENCE:}

[1] Hamilton DF. Bone Joint J 2014;96-B(5):622-8.

Acknowledgements: The c@h program is performed by Healthcare@home Deutschland $\mathrm{GmbH}$, funded by UCB Pharma, led by Elke Zeise and managed by Karen Thiel and Manh Dan Nguyen. We thank the patients and their caregivers in addition to the investigators and their teams who contributed to this program. Editorial services were provided by Costello Medical.

Disclosure of Interest: N. Böhme: None declared, A.-D. Holst Grant/research support from: AbbVie, BMS, Chugai, Pfizer, Paid instructor for: BMS, Lilly, UCB Pharma, F. Dybowski: None declared, C. Volberg: None declared, H.-G. Pott Paid instructor for: Hexal, Lilly, Novartis, MSD, Speakers bureau: AbbVie, Biogen, Chugai, Roche, Pfizer, Celgene, UCB Pharma, Medac, U. Lendl Employee of: UCB Pharma

DOI: 10.1136/annrheumdis-2018-eular.1989

\section{AB1261 DEVELOPMENT OF A LOCAL NEEDS-BASED GP CURRICULUM FOR SHARED CARE IN RHEUMATOLOGY}

O. Hussein, T. Duffy. Rheumatology, Connolly Hospital Blanchardstown, Dublin 15, Ireland

Background: There is a role for shared care between rheumatologist and primary care for Musculoskeletal (MSK) disorders. General practitioners (GPs) are involved in monitoring of clinical status of patients and their management, in addition to psychosocial aspects of chronic pain and disability. We are going to look at the rheumatology knowledge and skills needed by local GPs and based on their needs we plan to develop an initiative to improve the service provided to our patients. Here are the results of the initial survey of local GPs.

Objectives: We aim to identify knowledge and skill gaps in primary care related to MSK disorders and to develop a need-based curriculum to address these gaps. Develop an implementation plan to deliver the curriculum components.

Methods: Initially we identify the needs and gaps in the current practice using survey in a small group of local GPs. There are two rounds of questionnaires to reach a list of important areas to develop the curriculum. The second round will include large number of GPs. Also, there will be meetings with GPs to reach agreement and to adopt method of delivering these training and skills.

Results: In the first survey, 31 out of 36 GPs (86\%) returned questionnaires. One GP $(3.2 \%)$ refer to rheumatology department twice a week, 4 GPs $(13 \%)$ refe weekly, 23 GPs (74\%) refer every month and three GPs $(9.7 \%)$ rarely refer to rheumatologist. Reasons for referral were diagnosis and long-term management, ${ }^{21}$ diagnosis and discharge back to $\mathrm{GP}^{15}$ and patient's request. ${ }^{4}$ Thirteen GPs $(42 \%)$ have interest in rheumatology conditions management. Regarding GPs' concerns with rheumatology service, 22 of them (71\%) mentioned the long wait time for rheumatology appointment. Other issues were delayed clinic letter, need for clear instruction, patients not aware of appointment time, whether there is an urgent clinic and which clinic patient should be referred to (outpatient versus procedure clinics). Nineteen GPs (61\%) received undergraduate rheumatology training, $16(52 \%)$ during GP training programme and $4(13 \%)$ attended postgraduate courses. Topics of interest by GPs were variable rheumatology conditions in addition to joint injections, ${ }^{3}$ referral guidance ${ }^{2}$ and update on DMARD. Preferred teaching methods to deliver the syllabus were small group tutorials, ${ }^{12}$ face-to-face lectures $^{8}$ and protocols and guidelines. ${ }^{5}$

Conclusions: From this initial survey, we could identify that the major concern of local GPs is the long waiting time for rheumatology clinic. Some of GPs $(42 \%)$ expressed their interest to have further training in rheumatology. When we reach an agreed curriculum with GPs, we aim to deliver the syllabus through preferred methods by GPs. Shared care between rheumatologists and GPs would improve quality standard of care in community, provide patient care close to home and reduce referral rates. 